\title{
Comparison among Different Versions of Connor-Davidson Resilience Scale (CD-RISC) in Rehabilitation Patients after Unintentional Injury
}

\author{
Li Peng, Jiajia Zhang, Han Chen, Yu Zhang, Min Li ${ }^{\star}$, Yongju Yu and Botao Liu
}

Department of Military Psychology, School of Psychology, Third Military Medical University, Chongqing, China

*Corresponding author: Min Li, No.30, Gaotanyan Road, Shapingba District, Chongqing, China; Tel: 8602368771571; E-mail: limin52267@ tmmu.edu.cn

Received Date: July 17, 2014, Accepted Date: September 23, 2014, Published Date: September 30, 2014

Copyright: (c) 2014, Li Peng et al., This is an open-access article distributed under the terms of the Creative Commons Attribution License, which permits unrestricted use, distribution, and reproduction in any medium, provided the original author and source are credited.

\begin{abstract}
Objective: The current study was to compare the psychometric properties of the 25-item version (CD-RISC25), 10-item Connor-Davidson version (CD-RISC10) and 2-item Connor-Davidson version (CD-RISC2) in rehabilitation patients after injury.

Method: A sample of 115 Chinese patients was measured by CD-RISC25, CD-RISC10 and CD-RISC2, PTSD Checklist-Civilian Version (PCL-C) and Symptom Checklist-90-Revised (SCL-90-R) during the rehabilitation period.

Result: (1) Cronbach's alpha values indicated sufficient and high reliability to provide confidence in interpreting the score for the three versions; (2) The resilience measured by CD-RISC25 and CD-RISC10 both correlated negatively and significantly with PTSD and mental health problems, while this was not observed when resilience was measured by CD-RISC2; (3) Through ROC curves analyses, the CD-RISC25 and CD-RISC10 had similar discrimination for positive/negative group of SCL-90 and PTSD/non-PTSD.
\end{abstract}

Conclusion: The results supported the application of CD-RISC10 in clinical researches due to its excellent psychometric properties and time efficiency.

Keywords: Resilience; Rehabilitation; PTSD; Mental health problems; Unintentional injury

\section{Introduction}

Unintentional injuries such as car accidents, fights, industrial injury and natural disasters almost occur daily in our life. Previous researches reported that many people who had experienced catastrophic events may exhibit physical disability accompanied by certain psychological problems, such as depression, phobia and posttraumatic stress disorder (PTSD) $[1,2]$. It was found that up to $38 \%$ of injured patients were depressed about 6-12 months after injury [3,4]. Ryb et al. [5] also reported that injured patients were more likely to commit suicide compared with general population, and PTSD was more common after physical injuries, with $10 \%$ to $22 \%$ of injured patients [6,7]. Epidemiological investigation showed PTSD occurs in approximately $40 \%$ of people suffering car accidents, $78.6 \%$ suffering explosion accidents and $47 \%$ suffering sexual trauma in Chinese population [ 8 , 9].

However, some rehabilitative patients may cope well with negative events and pull through after injuries. This was due to the individuals' ability to bounce back that defined as resilience. Resilience was an indicator of good adjustment in the face of trauma, loss and other adverse life events [10,11]. Resilient and hardy characteristics were associated with better physical and mental health outcomes, more positive adaptive behaviors to negative life events [12]. Therefore, many researchers engaged in the studies of resilience and rehabilitation $[1,13]$. Quale and Schanke [14] identified three trajectories of psychological adjustment of eighty in patients with a severe injury at a rehabilitation hospital. Classification into the three trajectories was based on symptoms of psychological distress (posttraumatic stress disorder, depression, anxiety, and negative affect) and participants' level of positive affect at admission to and discharge from the rehabilitation hospital. Finally, the results indicated that most common trajectory was the resilience trajectory (54\%), followed by the recovery trajectory $(25 \%)$ and the distress trajectory (21\%). An adaptation pattern characterized by resilience was found to be the most common response to an acquired severe injury.

Furthermore, Bonanno and his colleagues [15] investigated 233 participants' longitudinal trajectories of depression and anxiety symptoms following spinal cord injury (SCI), which found that the majority of SCI patients demonstrated considerable psychological resilience.

Nevertheless, Resilience is a complex construct that is difficult to define and to measure, and the dearth of measuring methods may restrict empirical studies on resilience. Although in the past few years, there was an increase in the number of measures to assess resilience. Researchers developed scales that specifically measure resilience including the Resilience Scale [16], the Ego Resilience Scale [17], the Connor-Davidson Resilience Scale [12] and the Resilience Scale for Adults [18].

Among these measures, Connor-Davidson Resilience Scale was the most widely used scale till now [12]. It was initially applied in general population, such as primary care patients, psychiatric outpatients and PTSD samples, with good internal consistency, test-retest reliability, convergent and divergent validity. The 5-point Likert scale consists of 25 items using a ranging from 0 (not true at all) to 4 (true nearly all of the time), with higher scores reflecting higher resilience. Afterwards, some researchers began to explore a short version of CD-RISC 
considering the time efficiency. In 2007, a briefer version of CDRISC-10 was developed which addressed resilience as a unitary dimension [19]. CD-RISC10 was derived on the basis of exploratory and confirmatory factor analyses in large student samples; its shorter length offers a more homogeneous factorial basis and less respondent burden while providing comparable information as the longer scale (e.g. the 25 -item and 10-item scales correlate $>0.90$ ). Results from 1,743 undergraduate students indicated good internal consistency and construct validity of the measure. However, in contrast with the bulk of previous researches that have been performed with the assessment of CD-RISC25, there still have been limited publications based on the CD-RISC10, though the application of CD-RISC10 is increasing. Campbell-Sills et al. [20] studied demographic and childhood environmental predictors of resilience in a community sample, and offered new information about demographic and environmental correlates of self-reported resilience measured by CD-RISC10 in a large community sample. In addition, psychometric properties of the CD-RISC10 were examined in Chinese earthquake victims. The conclusion was that the Chinese version of the CD-RISC10 had excellent psychometric properties, and was applicable for Chinese people [21]. In addition, Vaishnavi et al. [22] had introduced a two item version of the longer CD-RISC. Like its longer originator, the two-item scale showed good test-retest reliability, convergent validity, and divergent validity. Further, the CD-RISC2 had significant correlation with the overall CD-RISC score as well as with each item of the CD-RISC, suggesting that the two items of the CD-RISC2 were good representatives of the overall scale and the CD-RISC2 could be used in lieu of the CD-RISC. However, there was no study to compare the psychometric properties of different versions of CD-RISC, especially in clinical patients.

Given the background above, the present study aimed at examining the psychometric properties and verifying the applicability of the three versions for assessing resilience in clinical patients during the rehabilitation period after trauma. We expect to find a resilience measure not only have its high psychometric properties but also show much time efficiency.

\section{Methods}

\section{Respondents and procedures}

The study was approved by the ethics committee of the 3 hospitals and all participants completed the informed consent process including verbal and written informed consent. 136 participants were recruited from rehabilitation inpatient clinics in 3 hospitals in Chongqing, China. The participants were asked to complete a separate response booklet with structured, anonymous, and self-reported questionnaires. Participants were carefully selected using stringent inclusion and exclusion criteria. The inclusion criteria for the participants are as follows: (1) all participants had experienced severe accidental injuries; (2) able to read and understand the questionnaires; (3) no history of traumatic brain injury (TBI) and consciousness disorder. Data from 21 participants including 11 patients with paraplegia and 10 with multiple fractures were excluded because these participants failed to respond to all of the items on the survey. The final sample comprised 31 females (26.9\%) and 84 males $(73.1 \%)$, with the age ranged from 14 to 64 $(\mathrm{M}=38.73, \mathrm{SD}=11.58)$. Taken thedemographic variables into account, there were 48 participants with high school diploma, 40 with bachelor's degree, 22 with master's degree, and 5 with doctoral degree. 63 patients were employed and 52 were not. With regard to marital status, 94 were married (81\%) and 21 were never married (19\%). There are 30 patients with paraplegia, 26 with multiple fractures, 32 with pelvic fractures and 27 with peripheral nerve injuries, which didn't affect the limbs motor function, and could write and respond to the questions.

\section{Measures}

Connor-Davidson Resilience Scale (CD-RISC): The CD-RISC is a 25-item 5-point Likert type assessment which measures the ability to cope with stress and adversity. Responses were made on a ranging from 0 (not true at all) to 4 (true nearly all the time) [12].The CDRISC has demonstrated adequate internal consistency, test-retest reliability, and convergent and divergent validity in general population and patient samples. Total scores range from 0 to 100, with higher scores reflecting greater resilience. To improve the read ability among Mainland Chinese, translations and back-translations were made by $\mathrm{Yu}$ and Zhang. The Chinese version demonstrated good psychometric properties [23].

10-item Connor-Davidson Resilience Scale (CD-RISC10): The new 10-item CD-RISC was extracted from the original 25-item CD-RISC, which includes item $1,4,6,7,8,11,14,16,17$ and 19. Respondents rated items on a scale from 0 (not true at all) to 4 (true nearly all the time). The CD-RISC10 is uni-dimensional and displays good internal consistency, discriminant validity and construct validity [19].

2-item Connor-Davidson Resilience Scale (CD-RISC2): Two items from the CD-RISC were used (the CDRISC2), namely items 1 ("Able to adapt to change") and 8 ("Tend to bounce back after illness or hardship"). These items were selected by the originators of the scale as etymologically capturing the essence of resilience, i.e., the ability to spring back and successfully adapt to change. Respondents rated items still on a scale from 0 (not true at all) to 4 (true nearly all the time). The CD-RISC2 has sound internal consistency, test-retest reliability, convergent validity, and divergent validity [22].

PTSD Checklist-Civilian version (PCL-C): The PCL is a 17 -item self-report checklist based on the DSM-IV diagnostic criteria for PTSD. Respondents rate the frequency of occurrence of each symptom on a 1-5 scale anchored by (Not at all) and (often) with an intermediate rating labeled (sometimes) [24]. Responses reflected symptoms resulting from any intrusive or traumatic events. The PCL$\mathrm{C}$ had been translated into Chinese and possessed good internal consistency, test-retest reliability, and convergent validity. The screening criterion of PTSD is over 38 points [25].

The Symptom Checklist-90-Revised (SCL-90-R): Respondents rate items on a 5-point scale reflecting their distress during the past 7 days. This 5-point Likert instrument is a multi-dimensional check list comprising 9 subscales (somatization, obsessive-compulsive, interpersonal sensitivity, depression, anxiety, hostility, phobia, paranoid ideation, and psychotics) [26]. The scale with good reliability and validity in Chinese sample has been validated and widely used in China, which can reflect individuals' mental health level effectively. Individual with total score exceeding 160 points were screened as positive referring to the DSM-IV [27].

\section{Statistical analysis}

Descriptive statistics were calculated on the three scales. In order to determine which scale of the three versions was much more closely correlated to PTSD and SCL-90, Pearson's correlation was conducted 
to study the criterion validity. ROC curves analyses were used to examine the discrimination for PTSD/non-PTSD and positive/ negative patients of SCL-90. SPSS software version 18.0 was performed for data analyses and $\mathrm{p}<0.05$ (two-tailed) was considered statistically significant.

\section{Result}

\section{Reliability and validity of the three versions of CD-RISC}

The reliabilities of the three versions were evaluated with internal consistency (Cronbach's alpha) [28]. The alpha value of CD-RISC25, CD-RISC10 and CD-RISC2 were 0.93, 0.88 and 0.76, respectively. For the three versions, Cronbach's alpha values indicated sufficient and high reliability to provide confidence in interpreting the score.

\section{Criterion validity of the three versions}

The correlations of resilience (measured by the three versions) with PTSD and SCL-90 could be viewed in Table1. There existed significantly negative relationship between resilience (measured by CD-RISC25 and CD-RISC10) and PTSD or mental health problems $(\mathrm{p}<0.01)$. The two versions both showed acceptable and good criterion validity. However, there were no significant correlations when resilience was measured by CD-RISC2 ( $>00.05)$.

\begin{tabular}{|l|l|l|}
\hline & PTSD & SCL-90 \\
\hline Resilience(CD-25 items) & $-0.506^{* *}$ & $-0.466^{* *}$ \\
\hline Resilience (CD-10 items) & $-0.531^{* *}$ & $-0.479^{* *}$ \\
\hline Resilience (CD-2 items) & -0.365 & -0.327 \\
\hline
\end{tabular}

Table 1: The relationships among resilience measured by three versions, PTSD and SCL-90( $n=115) .{ }^{*} \mathrm{p}<0.05 ;{ }^{* *} \mathrm{p}<0.01$

\section{Discrimination for SCL-90 and PTSD of CD-RISC25 and CD-RISC10}

The results of ROC curves analyses were showed in Table2. No significant difference existed between the areas under CD-RISC25 and CD-RISC10 ROC curves ( $\mathrm{p}=0.806$ (for SCL-90), $\mathrm{p}=0.750$ (for PTSD), $\mathrm{p}>0.05$ ). The above data were represented graphically in Figure 1 and Figure 2. Table 2 displayed best cut-off point, the sensitivity and specificity for each of the 4 ROC curves.

\begin{tabular}{|l|l|l|l|l|l|}
\hline ROC Curve & $\begin{array}{l}\text { Best cut- } \\
\text { off Point }\end{array}$ & Sensitivity & Specificity & AUC & p value \\
\hline CD-RISC25/SCL-90 & 57.5 & 0.730 & 0.628 & 0.717 & \multirow{2}{*}{0.806} \\
\hline CD-RISC10/SCL-90 & 21.5 & 0.730 & 0.705 & 0.730 & \\
\hline CD-RISC25/PTSD & 45.5 & 0.578 & 0.914 & 0.764 & \multirow{2}{*}{0.750} \\
\cline { 1 - 5 } CD-RISC10/PTSD & 19.5 & 0.667 & 0.843 & 0.785 & \\
\hline
\end{tabular}

Table 2: The data of ROC curve analysis on each curve, ${ }^{*} \mathrm{p}<0.05$, logistic regression for comparing the curve areas between the ROC curves of CD-RISC25 and CD-RISC10.

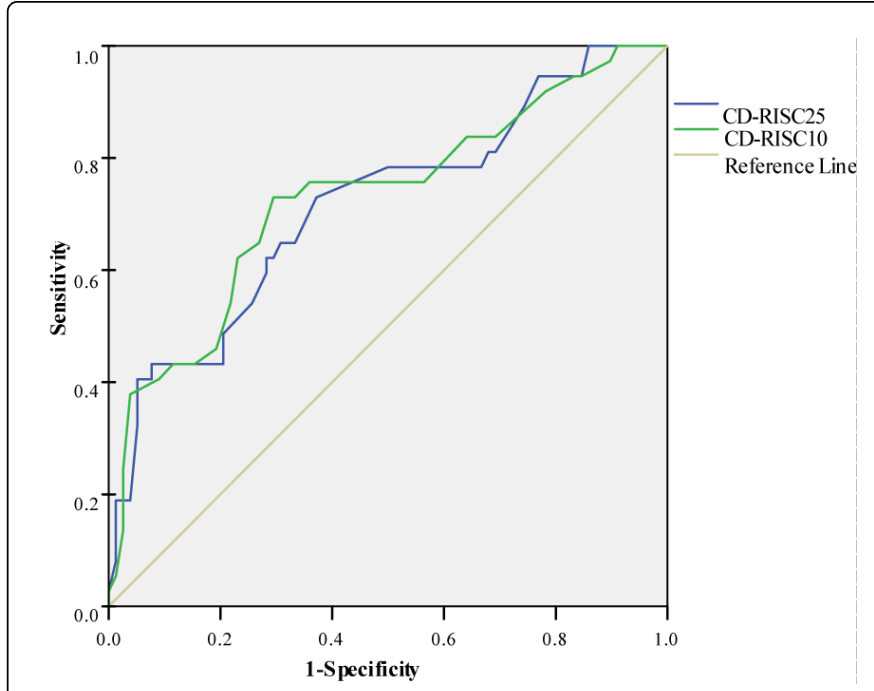

Figure 1: ROC curves of CD-RISC25 and CD-RISC10 for the sensitivity and 1-specificity of discriminating between positive and negative patients of SCL-90.

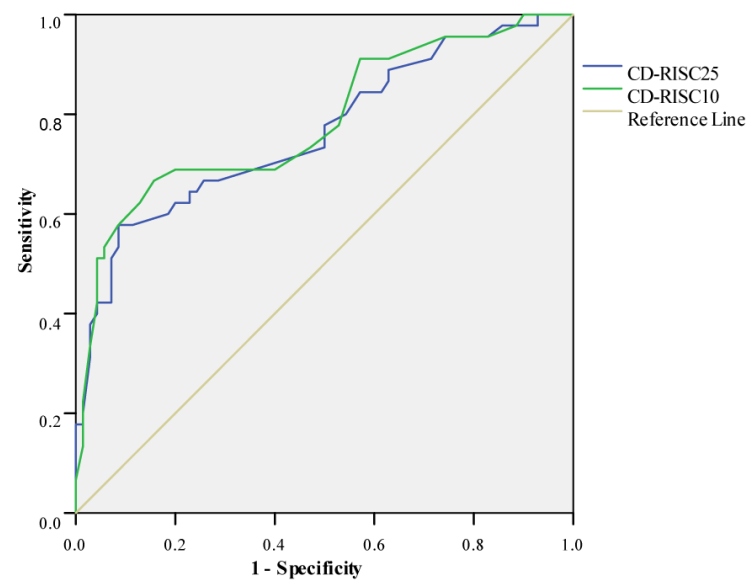

Figure 2: ROC curves of CD-RISC25 and CD-RISC10 for the sensitivity and 1-specificity of discriminating between patients with PTSD and non-PTSD.

\section{Discussion}

This study aimed to compare the 2-item, 10-item and 25-item versions of Connor-Davidson Resilience Scale in a sample of Chinese patients undergoing rehabilitation after injuries. Results showed that CD-RISC10 and CD-RISC25 had superior psychometric properties when compared with CD-RISC2. However, a shortened version may be more beneficial because of saving time. Thus, the CD-RISC10 version could be a satisfactory and convenient alternative for use in clinical researches.

The study found that the internal consistency was satisfied for each version of CD-RISC. For the three versions, Cronbach's alpha values indicated sufficient and high reliability to provide confidence in interpreting the score. Previous researchers had reported similar 
Page 4 of 5

results of the psychometric properties of the scales [21]. Criterion validities of the three versions were evaluated in the current study. Resilience scores measured by CD-RISC10 and CD-RISC25 correlated with PTSD or mental health problems negatively and significantly. The results were congruent with the previous literature that showed resilience buffered mental health problems such as depressive symptoms [29,30]. In addition, White et al. [31] assessed predictors of resilience in a trauma survivor sample, and their results indicated that there were significant correlations among resilience, satisfaction with life, spirituality, and depressive symptoms. However, there were no significant correlations when resilience was measured by CD-RISC2 in our research, which was different with the study results of Vaishnavi et al. [22]. They reported that CD-RISC2 was significantly relative to PTSD, and it may have predictive utility as a screen for PTSD and is sensitive to response to PTSD treatment.

The receiver operating characteristic curve was used to determine the performance of CD-RISC25 and CD-RISC10. The area under an ROC curve is a reasonable summary of the overall responsiveness of the measure. With the two versions of CD-RISC applying to the same group of patients, the larger the area under the curve of the measure, the greater the measure's consistency with the judgments as the positive group. The best cut point of the four curves can be a reference to differentiate patients from healthy, but the value was limited by the small sample. In addition, the sensitivity and the specificity of the curves were similar. The AUC (Area under Curve) of CD-RISC10 appeared better than that of the CD-RISC25, but there was no significant difference between them through analyses. In short, the two versions of CD-RISC demonstrated good discrimination for SCL-90 and PTSD. The results agreed with the study in Chinese earthquake victims [19]. All showed that to some extent the resilience evaluation measured by CD-RISC10 could differentiate people with PTSD or Psychotic symptoms from healthy.

\section{Limitations}

There are several limitations that should be noted in this study. First, all of the instruments that were utilized consisted of self-report measures, which may call into question the possibility of a response bias. Second, the current sample is relatively small, which needs to enlarge the sample in the future to better verify the results. In addition, our results should be replicated in other clinical or non-clinical populations especially the populations outside of China.

\section{Conclusions}

Despite these limitations, our study found that CD-RISC10 was a more appropriate instrument considering its excellent psychometric properties and time efficiency compared with CD-RISC25 and CDRISC2. On this account, the brief measure of resilience had much more applicability and usage in clinical researches. In the future study, CD-RISC10 should be put into use in clinical resilience survey.

\section{Acknowledgements}

We sincerely thank Jonathan R.T. Davidson and Kathryn M. Connor for providing us with original measure of Connor-Davidson Resilience Scale. We are grateful for the generous contributions of the research participants and the staff who assisted with data collection during the study. This research was supported by the National Natural Science Foundation of China (31170994) and National Social Science Fund of China granted (No. 14CSH072).

\section{References}

White B, Driver S, Warren AM (2008) Considering Resilience in the 1. Rehabilitation of People with Traumatic Disabilities. Rehabil Psychol 53: 9-17.

2. Zatzick DF, Marmar CR, Weiss DS, Browner WS, Metzler TJ, et al. (1997) Posttraumatic stress disorder and functioning and quality of life outcomes in a nationally representative sample of male Vietnam veterans. Am J Psychiatry 154: 1690-1695.

3. Michaels AJ, Michaels CE, Smith JS, Moon CH, Peterson C, et al. (2000) Outcome from injury: general health, work status, and satisfaction 12 months after trauma. J Trauma 48: 841-848.

4. Holbrook TL, Anderson JP, Sieber WJ, Browner D, Hoyt D (1998) Outcome after major trauma: discharge and 6 month follow-up results from the trauma recovery project. J Trauma-Injury Infection \& Critical Care 45: 315-324.

5. Ryb GE, Soderstrom CA, Kufera JA, Dischinger P (2006) Longitudinal study of suicide after traumatic injury. J Trauma 61: 799-804.

6. O'Donnell ML, Creamer M, Pattison P, Atkin C (2004) Psychiatric morbidity following injury. Am J Psychiatry 161: 507-514.

7. Davis RG, Ressler KJ, Schwartz AC, Stephens KJ, Bradley RG (2008) Treatment barriers for low-income, urban African Americans with undiagnosed posttraumatic stress disorder. J Trauma Stress 21: 218-222.

8. Chen H, Zhang JJ, Li M, Xu Y, Miao Y, Wang Q, Liu HL (2006) Correlation of prevalence of post traumatic stress disorder with mental health and resilience in rehabilitation patients with traumatic injury. Acta Academiae Medicine Militaris Tertiae 32: 2006-2009.

9. Xu W, Dong HB, Hu G, Song Y, Liang AM (2005) Post-traumatic stress disorder in the survivors of the tremendous explosion. Chinese J Clinical Rehabil 9: 238-241.

10. Rutter M (1985) Resilience in the face of adversity. Protective factors and resistance to psychiatric disorder. Br J Psychiatry 147: 598-611.

11. Rutter M (1990) Psychosocial resilience and protective mechanisms. Risk and protective factors in the development of psychopathology. Cambridge University Press.

12. Connor KM, Davidson JRT (2003) Development of a new resilience scale: The Connor-Davidson Resilience Scale (CD-RISC). Depress Anxiety 18: 76-82.

13. Quale AJ, Schanke AK (2010) Resilience in the face of coping with a severe physical injury: a study of trajectories of adjustment in a rehabilitation setting. Rehabil Psychol 55: 12-22.

14. Quale AJ, Schanke AK (2010) Resilience in the face of coping with a severe physical injury: a study of trajectories of adjustment in a rehabilitation setting. Rehabil Psychol 55: 12-22.

15. Bonanno GA, Kennedy P, Galatzer-Levy IR, Lude P, Elfström ML (2012) Trajectories of resilience, depression, and anxiety following spinal cord injury. Rehabil Psychol 57: 236-247.

16. Wagnild GM, Young HM (1993) Development and psychometric evaluation of the Resilience Scale. J Nurs Meas 1: 165-178.

17. Block J, Kremen AM (1996) IQ and ego-resiliency: conceptual and empirical connections and separateness. J Pers Soc Psychol 70: 349-361.

18. Friborg O, Hjemdal O, Rosenvinge JH, Martinussen M (2003) A new rating scale for adult resilience: what are the central protective resources behind healthy adjustment? Int J Methods Psychiatr Res 12: 65-76.

19. Campbell-Sills L, Stein MB (2007) Psychometric analysis and refinement of the Connor-davidson Resilience Scale (CD-RISC): Validation of a 10item measure of resilience. J Trauma Stress 20: 1019-1028.

20. Campbell-Sills L, Forde DR, Stein MB (2009) Demographic and childhood environmental predictors of resilience in a community sample. J Psychiatr Res 43: 1007-1012. 
Citation: Peng L, Zhang J, Chen H, Zhang Y, Li M, et al. (2014) Comparison among Different Versions of Connor-Davidson Resilience

Page 5 of 5

21. Wang L, Shi Z, Zhang Y, Zhang Z (2010) Psychometric properties of the 10-item Connor-Davidson Resilience Scale in Chinese earthquake victims. Psychiatry Clin Neurosci 64: 499-504.

22. Vaishnavi S, Connor K, Davidson JR (2007) An abbreviated version of the Connor-Davidson Resilience Scale (CD-RISC), the CD-RISC2: psychometric properties and applications in psychopharmacological trials. Psychiatry Res 152: 293-297.

23. Yu X, Zhang J (2007) Factor analysis and psychometric evaluation of the Connor-Davidson Resilience Scale (CD-RISC) with Chinese people. Soc Behav Pers 35: 19-30.

24. Weathers FW, Huska JA, Keane TM (1991) The PTSD Checklist-Civilian Version (PCL-C). Boston, Mass: National Center for PTSD.

25. Yu X, Zhang J (2007) A comparison between the Chinese Version of EgoResiliency Scale and Connor-Davidson Resilience Scale. Psychol Sci 30: 5.

26. Derogatis LR (1983) SCL-R: Administration, Scoring and Procedures Manual for the Revised Version, 1st Edition. Clinical Psychometric Research, Towson, MD.
27. Chen CH (1993) Symptom Checklist (SCL-90). J Chinese Ment Health, 7: 31-35 (Chinese article).

28. Cronbach LJ (1990) Essentials of psychological testing. New York: HarperCollins.

29. Peng L, Zhang J, Li M, Li P, Zhang Y, et al. (2012) Negative life events and mental health of Chinese medical students: the effect of resilience, personality and social support. Psychiatry Res 196: 138-141.

30. Pietrzak RH, Johnson DC, Goldstein MB, Malley JC, Rivers AJ, et al. (2009) Psychosocial buffers of traumatic stress, depressive symptoms, and psychosocial difficulties in veterans of Operations Enduring Freedom and Iraqi Freedom: The role of resilience, unit support, and post deployment social support. J Affect Disord 120: 188-192.

31. White B, Driver S, Warren AM (2010) Resilience and indicators of adjustment during rehabilitation from a spinal cord injury. Rehabil Psychol 55: 23-32. 